ARTICLE

https://doi.org/10.1038/s41467-018-08071-3

\title{
Altered chemistry of oxygen and iron under deep Earth conditions
}

Jin Liu (iD) 1,2, Qingyang Hu(D) 1, Wenli Bi ${ }^{3,4}$, Liuxiang Yang (1) 1,5, Yuming Xiao ${ }^{6}$, Paul Chow ${ }^{6}$, Yue Meng ${ }^{6}$ Vitali B. Prakapenka7, Ho-Kwang Mao, ${ }^{1,5} \&$ Wendy L. Mao ${ }^{2,8}$

A drastically altered chemistry was recently discovered in the Fe-O-H system under deep Earth conditions, involving the formation of iron superoxide $\left(\mathrm{FeO}_{2} \mathrm{H} x\right.$ with $x=0$ to 1$)$, but the puzzling crystal chemistry of this system at high pressures is largely unknown. Here we present evidence that despite the high $\mathrm{O} / \mathrm{Fe}$ ratio in $\mathrm{FeO}_{2} \mathrm{Hx}$, iron remains in the ferrous, spinpaired and non-magnetic state at $60-133 \mathrm{GPa}$, while the presence of hydrogen has minimal effects on the valence of iron. The reduced iron is accompanied by oxidized oxygen due to oxygen-oxygen interactions. The valence of oxygen is not -2 as in all other major mantle minerals, instead it varies around -1 . This result indicates that like iron, oxygen may have multiple valence states in our planet's interior. Our study suggests a possible change in the chemical paradigm of how oxygen, iron, and hydrogen behave under deep Earth conditions.

\footnotetext{
${ }^{1}$ Center for High Pressure Science and Technology Advanced Research, Beijing 100094, China. ${ }^{2}$ Department of Geological Sciences, Stanford University, Stanford, CA 94305, USA. ${ }^{3}$ Advanced Photon Source, Argonne National Laboratory, Argonne, IL 60439, USA. ${ }^{4}$ Department of Geology, University of Illinois at Urbana-Champaign, Urbana, IL 61801, USA. ${ }^{5}$ Geophysical Laboratory, Carnegie Institution of Washington, Washington, DC 20015, USA. ${ }^{6}$ HPCAT, X-Ray Science Division, Argonne National Laboratory, Argonne, IL 60439, USA. 7 Center for Advanced Radiation Sources, University of Chicago, Chicago, IL 60439, USA. ${ }^{8}$ Stanford Institute for Materials and Energy Sciences, SLAC National Accelerator Laboratory, Menlo Park, CA 94025, USA. Correspondence and requests for materials should be addressed to Q.H. (email: qingyang.hu@hpstar.ac.cn) or to H.-K.M. (email: hmao@gl.ciw.edu) or to W.L.M. (email: wmao@stanford.edu)
} 
$\mathrm{O}$ xygen and iron are Earth's most abundant elements by number of atoms and by mass, respectively. They form compounds dictating major chemistry of our planet ${ }^{1}$. It is conventionally accepted that the oxygen anion has an unvarying -2 valence state in mantle ferropericlase and bridgmanite throughout the deep interior, where the oxygen fugacity decreases with increasing depth. The redox states are mostly controlled by the $3 d$ transition element Fe which could vary among three valence states, metallic $\mathrm{Fe}^{0}$, ferrous $\mathrm{Fe}^{2+}$, and ferric $\mathrm{Fe}^{3+}$. Recently, a series of new iron oxides have been found with varying $\mathrm{O} / \mathrm{Fe}$ stoichiometry ranging from the endmember $\mathrm{Fe}^{3+}{ }_{2} \mathrm{O}_{3}$ on our planet's highly oxidized surface to the other end-member $\mathrm{Fe}^{2+} \mathrm{O}$ which should be stable at the highly reduced conditions in the deep lower mantle down to the coremantle boundary, which include $\mathrm{Fe}_{5} \mathrm{O}_{7}, \mathrm{Fe}_{4} \mathrm{O}_{5}$, and $\mathrm{Fe}_{5} \mathrm{O}_{6}$ (refs. ${ }^{2-4}$ ). High pressures in the deep lower mantle would promote the crystal field splitting of $3 d$ orbitals of iron and cause the electronic spin-pairing transition, which can affect the physical, chemical, and transport properties of mantle phases $^{5-7}$.

The conventional wisdom, however, is facing a change in light of the recent discovery of the high-pressure pyrite-structured iron superoxide $\mathrm{FeO}_{2}$ which has $\mathrm{O} / \mathrm{Fe}$ ratio even higher than $\mathrm{Fe}_{2} \mathrm{O}_{3}$ and can hold a varying amount of hydrogen (denoted as "Py$\mathrm{FeO}_{2} \mathrm{H} x$ " with $x$ from 0 to 1$)^{8-12,13-15}$. With the subducted plate carrying down water to react with the iron core to form Py$\mathrm{FeO}_{2} \mathrm{H} x$ and release hydrogen, oxygen-rich reservoirs could be accumulated in the very reducing core-mantle boundary region $^{13-15}$. Such reservoirs at the mid-point (2900 km depth) of the Earth's radius $(6370 \mathrm{~km})$ will certainly play a pivotal role in the global chemistry, including the generation of our present day aerobic atomosphere ${ }^{9,16}$. A number of key solid-state chemistry questions on $\mathrm{Py}-\mathrm{FeO}_{2} \mathrm{H} x$ must be understood: What are the valence states of $\mathrm{Fe}$ and $\mathrm{O}$ ? What is the nature of their chemical bonding? What are the effects of hydrogen on the valence and bonding of $\mathrm{Fe}$ and $\mathrm{O}$ ? Is the iron superoxide metallic? Are its $d$ electrons spin-paired?

Assuming oxygen in the common $\mathrm{O}^{2-}$ state as in other iron oxides, the valence state of iron would be ferryl $\left(\mathrm{Fe}^{4+}\right)$ for $\mathrm{FeO}_{2}$. On the other hand based on the analogy to $\mathrm{FeS}_{2}$ pyrite, in which Fe remains ferrous $\left(\mathrm{Fe}^{2+}\right)$ with the sulfur forming $\mathrm{S}_{2}{ }^{2-}$ dimer, would $\mathrm{FeO}_{2}$ also consist of $\mathrm{Fe}^{2+}$ cations and $\mathrm{O}_{2}{ }^{2-}$ dimer? Streltsov et al. ${ }^{17}$ predicted the valence state of iron to be +3 for the hydrogen-free $\mathrm{FeO}_{2} \mathrm{Py}$-phase where oxygen ions do not form $\mathrm{O}_{2}{ }^{2-}$ dimers, suggesting $\mathrm{FeO}_{2}$ "in between" the usual dioxides and peroxides, based on the density functional and dynamical mean-field theories (DFT + DMFT). By contrast, Jang et al. ${ }^{18}$ employed DFT + DMFT approaches with treating $\mathrm{FeO}_{2}$ to presumably have $\mathrm{O}_{2}{ }^{2-}$ dimer as a complete analogue of pyritestructured $\mathrm{FeS}_{2}$. Thus far, theoretical calculations cannot conclude the nature of chemical bonding and state of $\mathrm{FeO}_{2}$ without experimental constraints. Answers must be based on in situ measurements of the micron-sized $\mathrm{Py}-\mathrm{FeO}_{2} \mathrm{H} x$ sample which cannot be preserved after releasing pressure for normal electrochemical analysis.

We take the challenge by combining a variety of recently enabled synchrotron X-ray spectroscopic techniques ${ }^{19}$, and obtain intriguing answers from direct high-pressure experimentation supplemented by first-principles simulations with the self-consistent linear-response approach. Here we find many unexpected chemical behaviors in $\mathrm{Py}-\mathrm{FeO}_{2} \mathrm{H} x$ samples (Supplementary Figures 1-5), where iron is in the reduced, spin-paired ferrous state and the valence state of oxygen varies around -1 , instead of at -2 in common iron oxides, while hydrogen is weakly bonded in the structure. Our results suggest that other than iron, oxygen can occupy multiple valence states in our planet's interior, leading to a chemical paradigm change in the deep Earth.

\section{Results}

Valence states of iron and oxygen in $\mathrm{FeO}_{2} \mathrm{H} x$. The valence state of $\mathrm{Fe}$ can be characterized by near-edge X-ray absorption spectroscopy (XAS) features at energy slightly below the iron $K$-edge ${ }^{20}$. Because of the strong absorption of $7 \mathrm{keV} \mathrm{X}$-ray by diamond anvils, the XAS spectra were collected in the partial fluorescence yield (PFY) geometry for the iron superoxide at 53-133 GPa. That is, the incident X-ray beam passes through one diamond anvil, and then the X-ray fluorescence exits in the radial direction through the $\mathrm{Be}$ gasket to avoid double diamond absorption. The peak position and intensity distribution are diagnostic probes for valence state, spin state, geometry, and the ligand-ligand interactions for $\mathrm{Fe}$ atoms ${ }^{20}$. The iron $K$ pre-edge feature that is related to the $1 s \rightarrow 3 d$ electronic transition, is particularly sensitive to the valence state of iron ${ }^{21}$. Our XAS-PFY measurements of other representative iron compounds at ambient conditions explicitly show that the position of iron $K$ pre-edge absorption increases with increasing valence state (Fig. 1a top). The position of iron $K$ pre-edge of the iron superoxide $\mathrm{FeO}_{2}$ is very close, but slightly below, that of $\mathrm{Fe}^{2+}$ compounds. That is, the valence state of iron in $\mathrm{FeO}_{2}$ is plausibly close to +2 . Its position remains almost constant from 53 to $81 \mathrm{GPa}$, suggesting a minimal pressure dependence on the valence state of iron ${ }^{22}$, consistent with mantle ferropericlase and bridgmanite. Furthermore, it is intriguing that the iron valence state of the hydrogenfree iron superoxide is similar to that of the hydrogen-bearing Py$\mathrm{FeO}_{2} \mathrm{H} x$ with $x=0.5-0.7$ at $133 \mathrm{GPa}$. These observations indicate that the hydrogen content has minimal effects on the valence state of iron in the superoxide (Fig. 1a).

With the valence state of iron slightly under +2 , the valence state of oxygen for the $\mathrm{Py}-\mathrm{FeO}_{2}$ would have to be nearly -1 , suggesting that oxygen atoms must interact with each other in the iron superoxide. Such interaction can be observed experimentally from the oxygen $K$-edge spectroscopy. We note that the edge at $540 \mathrm{eV}$ is in a very soft X-ray region inaccessible to XAS in DAC experiments. Instead, we can access the oxygen $K$-edge by $\mathrm{X}$-ray Raman scattering (XRS) using hard X-ray in and hard X-ray out method, where the energy loss is measured due to inelastic Raman shift ${ }^{23,24}$. For XRS measurements on the oxygen $K$-edge, we deliberately used $\mathrm{LiF}$ as a pressure transmitting-medium, instead of water or $\mathrm{O}_{2}$, to assure XRS signals of oxygen only from the $\mathrm{Py}-\mathrm{FeO}_{2} \mathrm{H} x$ (Supplementary Figure 3). In the XRS at $110 \mathrm{GPa}$, we observed a sharp oxygen $\pi^{\star}$ peak near $531 \mathrm{eV}$ and a broad $\sigma_{u}{ }^{*}$ peak at $540-550 \mathrm{eV}$ (Fig. 1b). These peaks represent the $\mathrm{O}$ electron transition from $1 s$ core orbitals to the antibonding $1 \pi_{\mathrm{g}}{ }^{\star}$ and $3 \sigma_{u}^{*}$ orbitals, respectively (termed as $\pi^{\star}$ and $\sigma^{\star}$ transitions). The strong $\pi^{*}$ transition indicates the presence of the $\mathrm{O}-\mathrm{O}$ dimer.

Magnetic and electronic spin states of iron in $\mathrm{FeO}_{2} \mathrm{Hx}$. Magnetic ordering and spin state of $\mathrm{Fe}$ in iron superoxide can be investigated using synchrotron Mössbauer scattering (SMS) and $\mathrm{X}$-ray emission spectroscopy (XES), respectively. ${ }^{57} \mathrm{Fe}$ is a Mössbauer active isotope with nuclear resonant energy of 14.41 $\mathrm{keV}$. A series of SMS spectra of the iron superoxide were collected upon decompression in steps from $118 \mathrm{GPa}$ down to $32 \mathrm{GPa}$ (Fig. 2a), and the experiment was repeated in three separated runs (Supplementary Figures 6-8) of $\mathrm{FeO}_{2} \mathrm{H} x$ with different $x$ (Supplementary Table 1). SMS spectra were analyzed using CONUSS program $^{25}$ to extract the quadrupole splitting (QS) and isomer shift (IS) of iron in the Py-phase which is compared with that of standard samples of ferrous pyrite $\mathrm{FeS}_{2}$, ferrous $\mathrm{Fe}_{0.48} \mathrm{Mg}_{0.52} \mathrm{O}$, and ferric $\mathrm{Fe}_{2} \mathrm{O}_{3}$ (Fig. 2b). The QS of iron in the Py-phase barely 

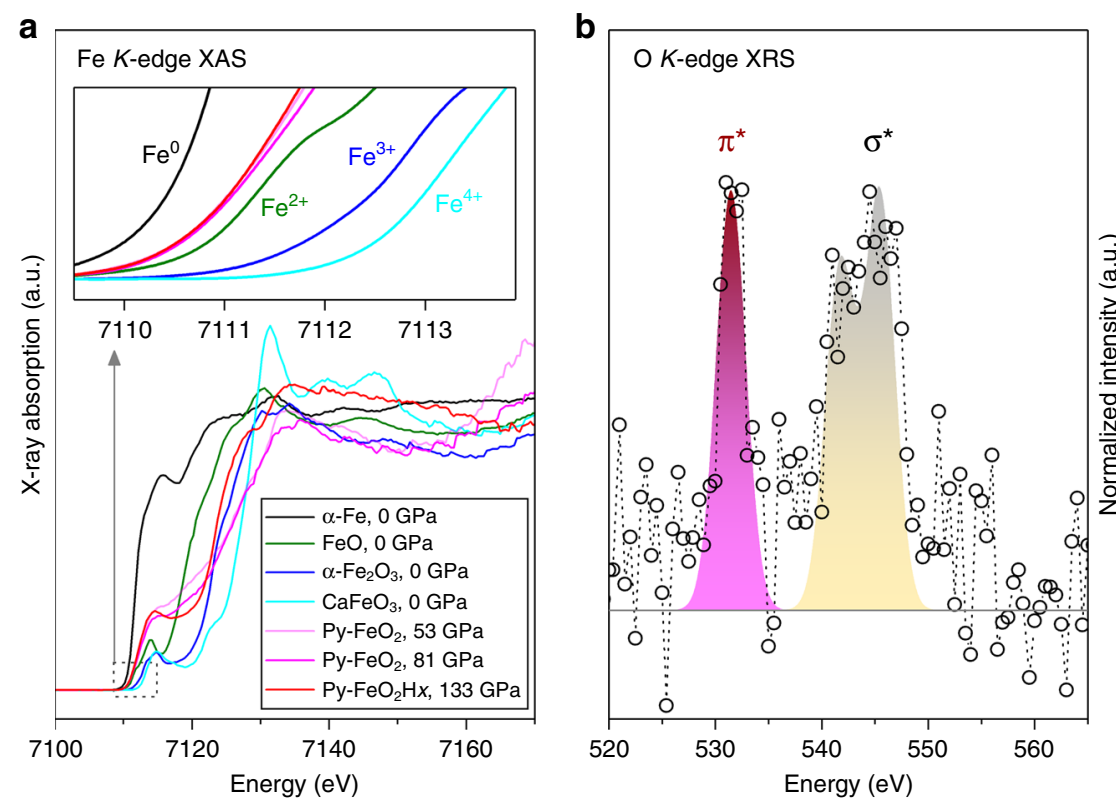

Fig. 1 Representative XAS and XRS spectra of iron oxide compounds. a PFY-XAS spectra at Fe K-edge of iron oxide compounds at room temperature. Black, olive, blue, and cyan lines: $\mathrm{Fe}^{0}, \mathrm{Fe}^{2+} \mathrm{O}, \mathrm{Fe}^{3+}{ }_{2} \mathrm{O}_{3}$, and $\mathrm{CaFe}^{4+} \mathrm{O}_{3}$ at ambient conditions, respectively; light and dark magenta lines: $\mathrm{Py}-\mathrm{FeO}$, at 53 and $81 \mathrm{GPa}$, respectively; red line: $\mathrm{Py}-\mathrm{FeO}_{2} \mathrm{Hx}$ at $133 \mathrm{GPa}$; gray arrow: the link from the dashed outline to the inset. Inset: the area zoomed for the dashed outline in Fig. 1a. b XRS spectra of $\mathrm{Py}-\mathrm{FeO}_{2} \mathrm{Hx}$ at $110 \mathrm{GPa}$. Circles: experimental data; shaded areas: fitted peaks

increased with increasing pressure, whereas the IS displayed an opposite trend. The pressure dependence of QS and IS for iron in the superoxide is comparable to that for ferrous iron $\left(\mathrm{Fe}^{2+}\right)$ in ferropericlase $(\mathrm{Mg}, \mathrm{Fe}) \mathrm{O}$ in the low-spin state (refs. $\left.{ }^{26,27}\right)$, likely due to the ferrous nature of the iron superoxide. It is noted that the spin transition could largely affect the QS of iron in $(\mathrm{Mg}, \mathrm{Fe})$ $\mathrm{O}$ (refs. ${ }^{27,28}$ ). Furthermore, the values of the QS and IS of iron in the superoxide extrapolated to ambient conditions are about 0.27 and $0.55 \mathrm{~mm} \mathrm{~s}^{-1}$, in excellent agreement with that in pyrite $\mathrm{FeS}_{2}$ (ref. ${ }^{29}$ ).

Our SMS spectra in Fig. 2a consistently showed that the Pyphase remained in the non-magnetic state represented by the clean, single beat spectra, regardless of the content of hydrogen in the lattice. SMS data with multiple time beats indicating magnetic ordering appeared only below $40-50 \mathrm{GPa}$ when the iron superoxide decomposed into a magnetic phase under decompression. We note that the full width at half maximum (FWHM) for the diffraction peaks for the iron superoxide broadened significantly below $65 \mathrm{GPa}$ and the peak intensity continually decreased until they finally disappeared around $40 \mathrm{GPa}$ upon decompression at room temperature. Furthermore, the QS and IS values of iron in the magnetic phase are close to that in hematite $\left(\mathrm{Fe}_{2} \mathrm{O}_{3}\right)$ at 32 $\mathrm{GPa}$. It is consistent with our recent X-ray diffraction (XRD) observations where the new diffraction peaks can be indexed to hematite below $40 \mathrm{GPa}$.

The magnetic spin states of $3 d$ electrons are also directly probed by XES spectra of the $\mathrm{Fe} K_{\beta}$ fluorescence lines ${ }^{5,30}$ of the iron superoxide between 40 and $133 \mathrm{GPa}$ (Fig. 3). The intensity of the $K_{\beta}^{\prime}$ satellite peak of iron in the superoxide decreased between 45 and $60 \mathrm{GPa}$ and disappeared completely at $60-65 \mathrm{GPa}$ where the total spin momentum $(S)$ of iron in the Py-phase approached zero, indicating that iron in the Py-phase undergoes a gradual electronic spin-pairing transition below $65 \mathrm{GPa}$ from high-spin to low-spin states (Supplementary Figure 9). We note that the XES spectra were collected with decreasing pressure and thus the spin transition pressure may be higher due to pressure hysteresis on the spin crossover upon decompression ${ }^{31}$. These observations also suggest that the iron superoxide is not stable in the high-spin state and would decompose into oxygen and hematite across the electronic spin transition of iron, associated with the valence change in oxygen and iron at the same time.

Oxygen bonding and charge transfer in $\mathrm{FeO}_{2}$ and $\mathrm{FeO}_{2} \mathrm{H}$. For pyrite-type structures, the covalent nature is mainly controlled by the anion-anion distance $\left(D_{O}\right)$. We followed previous strategies $^{32,33}$ and used the fractional coordination $y$ of oxygen to compare $\mathrm{O}-\mathrm{O}$ distance at different pressures:

$$
y=\frac{1}{2}-\frac{\sqrt{3} D_{0}}{6 a}
$$

where $a$ is the lattice parameter as a function of pressure. The archetypical pyrite $\mathrm{FeS}_{2}$ with $y=0.385$ forms solid anion dimers with divalent $\mathrm{Fe}$ cations at ambient conditions. In contrast, conventional dioxides with tetravalent cations (e.g. $\mathrm{RuO}_{2}$ and $\mathrm{OsO}_{2}$ ) have a smaller value of $0.34-0.35$ for $y$ in the pyrite-type structure $^{33}$. Our previous experimental XRD data showed $y=$ 0.371 with $D_{0}=1.937 \AA$ in the iron superoxide at $75 \mathrm{GPa}$ which lies between conventional dioxides and peroxides ${ }^{9,33}$. Due to the importance of the O-O bond length, we collected XRD patterns on a nearly pure $\mathrm{FeO}_{2}$ sample at $96 \mathrm{GPa}$, which have been further analyzed by using the Rietveld method for the primary phase of $\mathrm{FeO}_{2}$ and the LeBail method for the weaker $\mathrm{O}_{2}$ and $\mathrm{Fe}_{2} \mathrm{O}_{3}$ phases. The final refinement factors are $R_{1}=0.069$ and $w R_{2}=0.14$. The bond length $\left(D_{0}\right)$ for $\mathrm{FeO}_{2}$ is $\sim 1.87(1) \AA$ and the lattice parameter is $4.2855(3) \AA$ with $y=0.374$ at $96 \mathrm{GPa}$. Therefore, compared with various peroxides synthesized at ambient pressure ${ }^{34}$, the $\mathrm{O}-\mathrm{O}$ bonding length in the iron superoxide is relatively longer. With $0.35<y<0.39$, iron-oxygen octahedrons are chained by the $\mathrm{O}-\mathrm{O}$ bonding as shown in Fig. 4a.

We further conducted first-principles simulations (see Methods) to construct the charge density landscape at $100 \mathrm{GPa}$ (Fig. 4). Following the Bader charge division scheme, we determined the charge of the Fe atoms are 6.38 e by DFT $+U$ and 6.32 e by the 


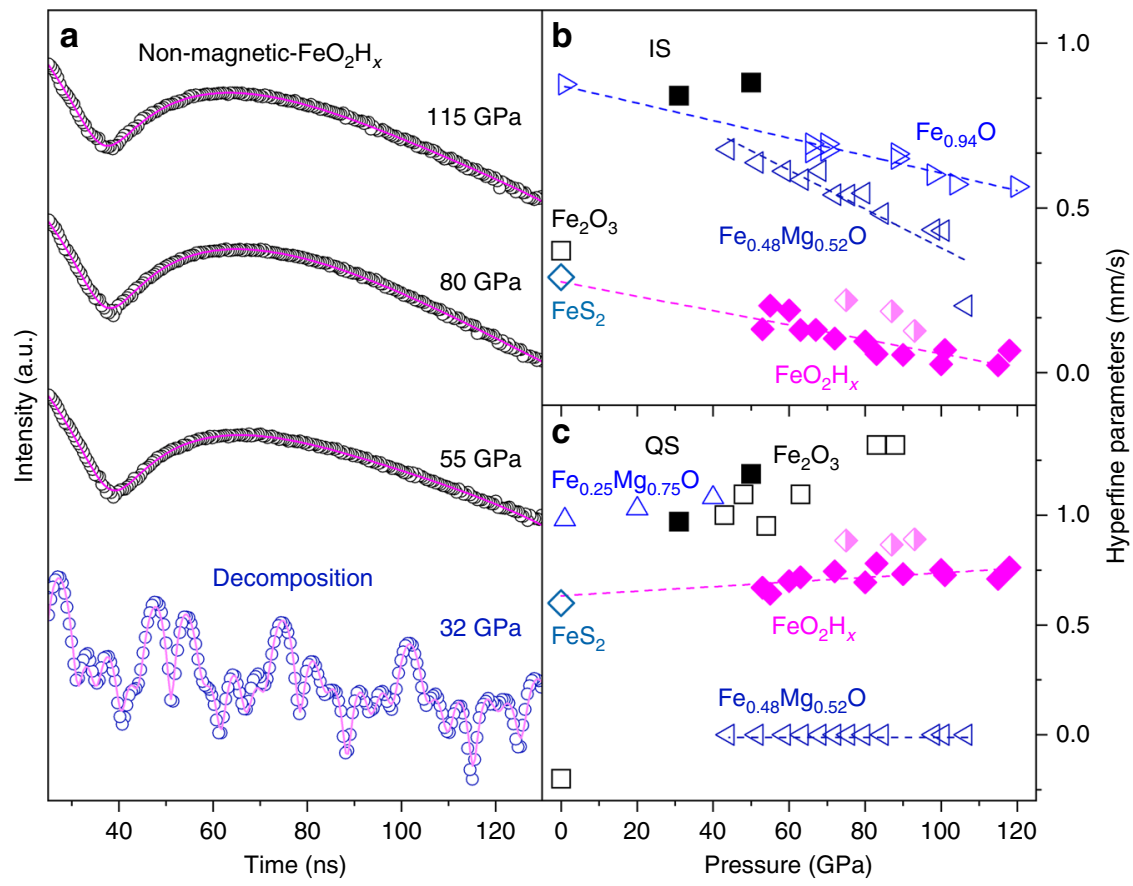

Fig. 2 Representative SMS spectra and hyperfine parameters. a High pressure Mössbauer spectra of $\mathrm{FeO}_{2} \mathrm{Hx}$ collected upon decompression at $300 \mathrm{~K}$. Circles: experimental SMS spectra; red solid lines: modeled spectra using CONUSS program. Comparison of isomer shift (b) and quadrupole splitting (c) of iron in iron-rich compounds at high pressure and room temperature. Solid and half-filled diamonds: pyrite-structured $\mathrm{FeO}_{2} \mathrm{H}$ and $\mathrm{FeO}{ }_{2}$, respectively, this study; open diamonds: pyrite $\mathrm{FeS}_{2}$ (ref. ${ }^{29}$ ); solid squares: $\mathrm{Fe}_{2} \mathrm{O}_{3}$, this study; open squares: $\mathrm{Fe}_{2} \mathrm{O}_{3}$ (ref. ${ }^{58}$ ); left-, right-, and top-pointing triangles: $\left(\mathrm{Fe}_{0.48} \mathrm{Mg}_{0.52}\right) \mathrm{O}$ (ref. ${ }^{27}$ ), $\mathrm{Fe}_{0.94} \mathrm{O}$ (ref. ${ }^{26}$ ), and $\left(\mathrm{Fe}_{0.25} \mathrm{Mg}_{0.75}\right) \mathrm{O}$ (ref. ${ }^{28}$ ), respectively; dashed lines: linear fits to experimental data. The errors on the experimental data are $\pm 2 \mathrm{SD}$, which are smaller than symbols and not shown for clarity

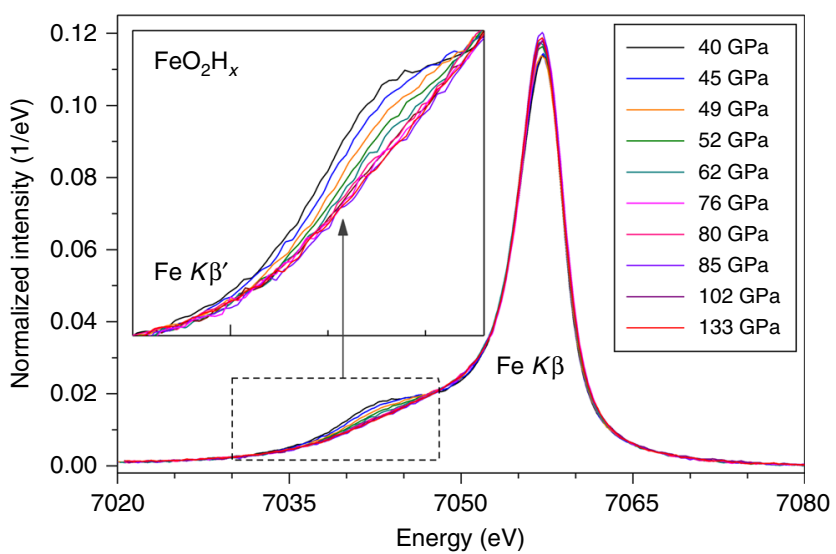

Fig. 3 High-pressure XES spectra of pyrite-structured $\mathrm{FeO}_{2} \mathrm{Hx}$ at $300 \mathrm{~K}$. The integrated intensity of the XES spectra was normalized to unity. Inset: the $K_{\beta}^{\prime}$ satellite emission peak between 7030 and $7048 \mathrm{eV}$ after the iron main emission peaks was aligned to that at $133 \mathrm{GPa}$. The changes of the satellite peak intensity in $\mathrm{FeO}_{2} \mathrm{H} x$ are attributed to the high-spin to low-spin transition, as the disappearance of the satellite peak has been used as a robust criterion for the electronic spin-pairing transition of iron in other iron-bearing compounds 30

Heyd-Scuseria-Ernzerhof (HSE) screened hybrid functional (screening variable of 0.15) free of the empirical Hubbard parameter $U$. Based on our experimental observations that Py$\mathrm{FeO}_{2} \mathrm{H} x$ is in the non-magnetic state, the $\mathrm{O}-\mathrm{O}$ bond length values from our GGA $+U$ calculations are consistent with our XRD experimental results as well as $\mathrm{Lu}$ et al. ${ }^{35}$. We note that the O-O bonding length is sensitive to computational and physical environments and that either antiferromagnetic or ferromagnetic configurations would yield a longer $\mathrm{O}-\mathrm{O}$ bond by $\sim 10 \%{ }^{35}$. The valence state of iron is $+1.62(\mathrm{DFT}+U)$ or +1.68 ( $\mathrm{HSE})$ and that of oxygen is -0.81 (DFT $+U$ ) or -0.84 (HSE) for $\mathrm{FeO}_{2}$ (Table 1). It is known that Bader analysis may underestimate the charge transfer between anions and cations ${ }^{36}$. Our calculations may not indicate the exact valence values, but clearly show charge transfer between anions, which has also been seen in the calculations of archetypal pyrite $\mathrm{FeS}_{2}$ (ref. ${ }^{37}$ ). In hydrogen-bearing $\mathrm{Py}^{-} \mathrm{FeO}_{2} \mathrm{H}$, hydrogen is equally bonded with two nearest oxygen atoms ${ }^{38}$. As a result, hydrogen is calculated to be +0.64 at $100 \mathrm{GPa}$. Oxygen atoms adopt electrons from hydrogen and become -1.13 while the valence state of iron remains around +1.63 for the $\mathrm{Py}-\mathrm{FeO}_{2} \mathrm{H}$. Therefore, hydrogen only donates electrons to oxygen atoms and has a minimal effect on the valence state of iron.

\section{Discussion}

Looking at individual properties, $\mathrm{FeO}_{2}$ and $\mathrm{FeO}_{2} \mathrm{H} x$ may appear similar to pyrite $\mathrm{FeS}_{2}$ in their crystal structure, cation and anion valences, and spin-pairing state. Assimilating the comprehensive experimental and theoretical investigations, however, points to fascinating changes of high-pressure crystal chemistry above $70 \mathrm{GPa}$ which corresponds to roughly the pressures beyond $1700 \mathrm{~km}$ depth, which is the midpoint separating the Earth's mass into two equal halves. At higher pressures, the valence of oxygen in oxides is no longer fixed to -2 , but becomes variable. The valence of iron becomes more dependent on the crystal structure and pressure rather than the oxygen stoichiometry. Hydrogen is no longer bonded to oxygen as $\mathrm{OH}$, but becomes mobile. $\mathrm{FeO}_{2}$ and $\mathrm{FeO}_{2} \mathrm{H} x$ do not exactly belong to the pyrite $\mathrm{FeS}_{2}$ type structure with interactive anion dimer, nor the $\mathrm{PdF}_{2}$ type structure without interactive anion dimer, but represent a new structure type in the $P a \overline{3} \mathrm{MA}_{2}$ family with 


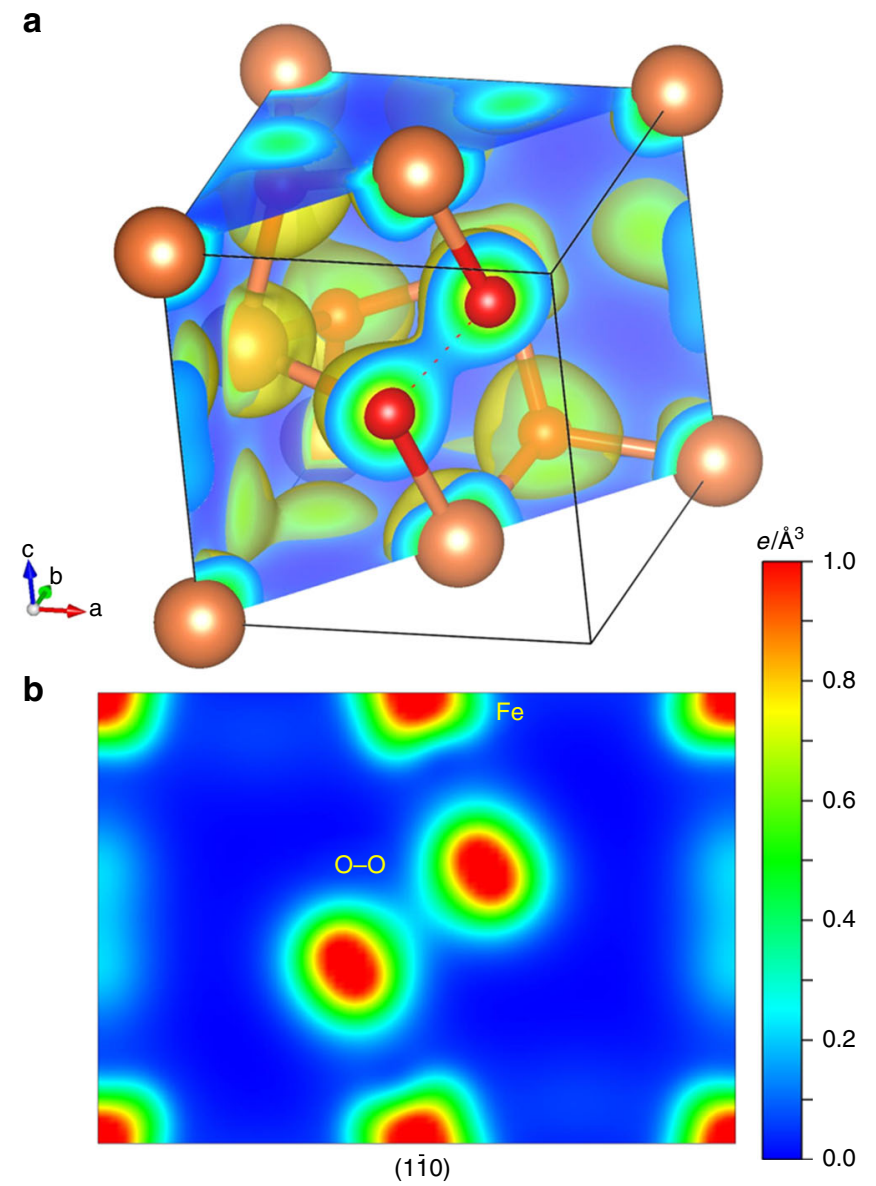

Fig. 4 The calculated charge density landscape of pyrite-structured $\mathrm{FeO}_{2}$ at $100 \mathrm{GPa}$. a Gold surface represents charge density of $0.15 \mathrm{e} / \AA^{3}$. The section cut through the $(1 \overline{1} 0)$ plane shows the weak $\mathrm{O}-\mathrm{O}$ bonding feature. Brown balls are Fe atoms and red balls are $\mathrm{O}$ atoms. b Charge density distribution projected on the (110) plane. The level of charge density is reflected by the color bar

partially interactive anion dimer. These issues have been previously noted as separate enigmas in other studies of $\mathrm{O}, \mathrm{Fe}$, and $\mathrm{H}$ under high pressures, such as formation of $\mathrm{O}_{8}$ molecules ${ }^{39}$, the redox paradox of ferric iron ${ }^{40}$, and mobility of hydrogen in ice $^{41,42}$. With the integrated study here, now the overall picture clearly indicates a chemical paradigm change under deep Earth conditions.

At zero pressure without external constraint, the $\mathrm{O}-\mathrm{O}$ interaction type can be empirically determined by their distance ${ }^{33,34}$, 1.29-1.53 $\AA$ for bonded dimers in peroxides and 2.45-2.72 $\AA$ for non-interactive oxygen in $\mathrm{PdF}_{2}$-type dioxides. Under pressures, however, the intermolecular interactions are further controlled by additional external forces that can generate additional intermolecular bonds. For instance, $\mathrm{O}_{2}$ transform to the high pressure $\varepsilon-\mathrm{O}_{8}$ phase with two types of bond length $D_{0}=1.20 \AA$ and $2.18 \AA$, respectively. The O-O dimer of $D_{0}=1.89 \AA$ (refs. ${ }^{24,39,43}$ ), the reduced valence of oxygen in $\mathrm{FeO}_{2}$ and $\mathrm{FeO}_{2} \mathrm{H} x$, and the formation of new type of $\mathrm{Pa} \overline{3}$ peroxide are a natural consequence of high-pressure chemistry.

At zero pressure, the valence of iron or ferric/ferrous ratio is generally correlated to the oxygen fugacity. The correlation is weakened by the additional control by the crystal structure of high-pressure phases. For instance, the paradoxical correlation of lower oxygen fugacity with higher $\mathrm{Fe}^{3+}$ abundance ${ }^{40}$ reflects the ability of the high-pressure perovskite structure to
Table 1 The valence state of $F e$ and $O$ atoms from the electron charge density of Py-phase at $100 \mathrm{GPa}$ and $0 \mathrm{~K}$

\begin{tabular}{lcc}
$\mathbf{F e O}_{\mathbf{2}}$ & DFT $+\mathbf{U}$ & HSE \\
\hline $\mathrm{Fe}$ & 1.62 & 1.68 \\
$\mathrm{O}$ & -0.81 & -0.84 \\
$y$ & 0.367 & 0.362 \\
\hline
\end{tabular}

accommodate more $\mathrm{Fe}^{3+}$ iron ${ }^{44}$. Here the new high-pressure iron superoxide structure is able to keep its iron in ferrous state by reducing the oxygen valence, and fits well in the reducing environment of the core-mantle boundary. The spin-paired non-magnetic $\mathrm{Fe}^{2+}$ also fits the high-pressure chemistry of the deep lower mantle ${ }^{5,30}$.

At zero pressure, hydrogen is present as $\mathrm{H}^{+}$and replaces other cations without affecting the anion valence. Here, the addition of hydrogen has negligible effects on the chemical and magnetic properties of the iron in $\mathrm{FeO}_{2} \mathrm{H} x$, but mainly affects the oxygen valence. This shows the change of hydrogen from strong $\mathrm{OH}$ covalent bond at low pressures to weak interaction in the $\mathrm{Py}-\mathrm{FeO}_{2} \mathrm{H} x$ lattice. Hydrogen chemistry in $\mathrm{H}_{2} \mathrm{O}$ is known to have a very important change at high pressures to become symmetrically hydrogen bonded at low temperature ${ }^{42}$ and superionic at high temperature ${ }^{41,45}$; both result in weakening the $\mathrm{OH}$ bonding. The present observation indicates the weakened interaction of hydrogen is not limited to $\mathrm{H}_{2} \mathrm{O}$ but is a general high-pressure chemical trend that has many important consequences, including loss of hydrogen in the formation of $\mathrm{Py}-\mathrm{FeO}_{2} \mathrm{Hx}$ (ref. ${ }^{38}$ ), spontaneous splitting of $\mathrm{H}_{2} \mathrm{O}$ into hydrogen and oxygen ${ }^{13}$, and separation of water and hydrogen cycles in the deep Earth ${ }^{10}$.

Our study demonstrates that under extreme pressures of the deep mantle, the structure type becomes a main controlling factor of valence states, and the conventional sense of valence of elements could be altered to fit the structure. The new type of structure has the unexpected valence state of oxygen that is different from the $\mathrm{O}^{2-}$ state in primary mantle oxides and silicates, and the ferrous, spin-paired, and non-magnetic state of iron that would affect $\mathrm{Mg}-\mathrm{Fe}$ partitioning between mantle phases ${ }^{46,47}$. We may expect the new iron superoxide in pyrite structure to accommodate and form solid solutions with other divalent and trivalent cations such as $\mathrm{Mg}^{2+}$ and $\mathrm{Al}^{3+}$ (ref. ${ }^{48}$ ) and with anions, such as sulfur and halogens. We may further expect that the $\mathrm{Pa} \overline{3}$ peroxide structure is only one example of the possible compositions in the deep Earth, and that additional structure types with different valence and spin, such as the recently discovered hexagonal phase ${ }^{48}$, will emerge.

\section{Methods}

Sample synthesis and characterization. The five samples of the pyrite-type phase have been synthesized and further probed by X-ray spectroscopic techniques in this study (Supplementary Table 1). Iron superoxide samples of $\mathrm{FeO}_{2}$ or $\mathrm{FeO}_{2} \mathrm{H} x$ were synthesized from goethite $(\mathrm{FeOOH})$ or ${ }^{57} \mathrm{Fe}$-enriched hematite $\left(\mathrm{Fe}_{2} \mathrm{O}_{3}\right)$ mixture with $\mathrm{O}_{2}$ and water $\mathrm{H}_{2} \mathrm{O}$, respectively, at $1800-2200 \mathrm{~K}$ under target pressures in laser-heated DACs at 16IDB, 13ID-D and High Pressure Synergetic Consortium (HPSynC) of the Advanced Photon Source of Argonne National Laboratory. In order to avoid any signal contamination from other phases in the sample and to assure complete transition, the whole starting materials of goethite $(\mathrm{FeOOH})$ or hematite $\left(\mathrm{Fe}_{2} \mathrm{O}_{3}\right)$ have been laser-heated repeatedly for $2-3 \mathrm{~h}$ at target pressures to synthesize a pure pyrite-structured $\mathrm{FeO}_{2} \mathrm{H} x(x=0-1)$ phase.

XRD patterns were collected with the mapping steps of $4 \mu \mathrm{m}$ for the wholesynthesized samples at beamlines 13ID-D or 16ID-B of Advanced Photon Source (APS), Argonne National Laboratory (ANL), confirming that the whole sample is the pyrite-type phase without any detectable amount of starting materials goethite or hematite left in the DACs (Supplementary Figures 1-5). The hydrogen content $(x)$ of the iron superoxide samples that were synthesized from $\mathrm{FeOOH}$ or $\mathrm{Fe}_{2} \mathrm{O}_{3}$ 
with $\mathrm{H}_{2} \mathrm{O}$ was estimated to be $0.5-0.8$ based on the unit-cell parameter calibration ${ }^{10}$ (Supplementary Table 1). In particular, a relatively small size of starting materials were selected to be $25-30 \mu \mathrm{m}$ in diameter with a thickness of 6-8 $\mu \mathrm{m}$, which can reduce the synthesis time. We note that the X-ray beam was focused down to $5-7 \mu \mathrm{m}$ in the full-width at half-maximum (FWHM) at the sample position. Thus the sample center was deliberately probed in order to achieve a relatively high efficiency of the spectroscopic measurements. That is, about one fifth of the volume of the synthesized samples was probed in the spectroscopic measurements.

Synchrotron X-ray diffraction experiments. XRD experiments were carried out at beamlines 13-IDD and 16-IDB of the Advanced Phonon Source, Argonne National Laboratory. A highly monochromatized incident X-ray beam was used with an energy of $30.49 \mathrm{keV}(0.3738 \AA), 33.17 \mathrm{keV}(0.3738 \AA)$, or $37.08 \mathrm{keV}(0.3344 \AA)$. The incident $\mathrm{X}$-ray beam reached the sample position with a beam size of $2-5 \mu \mathrm{m}$ in FWHM. For laser-heating XRD, two infrared laser beams were focused down to $\sim 15 \mu \mathrm{m}$ and $25-30 \mu \mathrm{m}$ in the flat top area of the heating profile on both sides of the sample at beamlines 13-IDD and 16-IDB, respectively. With the help of the X-ray induced luminescence on the sample and/or ruby, both laser beams were co-axially aligned with the incident $\mathrm{X}$-ray beam. The temperature of the samples was calculated through fitting the measured thermal radiation spectra with the gray-body approximation ${ }^{49,50}$. The temperature uncertainty is $100-200 \mathrm{~K}$. A tiny piece of gold was placed in the sample chamber and its lattice parameters of gold were applied to determine pressure and uncertainty ${ }^{51}$.

Synchrotron Mössbauer spectroscopy experiments. The SMS spectra of ${ }^{57} \mathrm{Fe}-$ enriched Py-phase and $\mathrm{Fe}_{2} \mathrm{O}_{3}$ samples were collected at beamline 16-IDD, APS, ANL. A monochromatic X-ray beam with an energy of $14.41 \mathrm{keV}$, a bandwidth of $2 \mathrm{meV}$, and $5-7 \mu \mathrm{m}$ in FWHM was used to excite the ${ }^{57} \mathrm{Fe}$ nuclei in the sample ${ }^{52}$. An avalanche photodiode detector (APD) was used to collect the timedelayed SMS signals in the forward direction with a typical collection time of $\sim 2-4 \mathrm{~h}$ for each pressure. Pressure before and after each measurement was determined based on the Raman spectra of the diamond anvils where the sample contacted ${ }^{53}$. Py-phase samples were synthesized from the assemblages of $\mathrm{Fe}_{2} \mathrm{O}_{3}$ and $\mathrm{H}_{2} \mathrm{O}$ or $\mathrm{O}_{2}$ approximately at $90-100 \mathrm{GPa}$ and $2000 \mathrm{~K}$. After the SMS spectrum of the sample was collected, a thin stainless-steel foil or a platelet of sulfate heptahydrate $\left(\mathrm{FeSO}_{4} \cdot 7 \mathrm{H}_{2} \mathrm{O}\right)$ was placed on the downstream side of the DAC to serve as a reference for the isomer shift measurements. Mössbauer hyperfine parameters, including quadrupole splitting, isomer shift, and magnetic hyperfine field of the samples were derived using the CONUSS program ${ }^{52}$.

X-ray absorption spectroscopy experiments. Why use XAS measurements, collected in partial fluorescence yield mode (XAS-PFY), were carried out at beamline 16-IDD, APS, ANL. A monochromatic X-ray beam of at $7112 \mathrm{eV}$ with $1 \mathrm{eV}$ bandwidth from a water-cooled diamond (111) double-crystal monochromator was focused down to 5-7 $\mu \mathrm{m}$ in FWHM at the sample position. The XAS-PFY spectra were collected by setting the Bragg angle of the $\mathrm{Si}$ (333) analyzer to the maximum of the $K \alpha_{1}$ emission line while scanning the incident energy with an energy step of $0.4 \mathrm{eV}$ through the Fe $K$ pre-edge between 7086 and $7186 \mathrm{eV}$ (Fig. 2a). The XAS-PFY spectra of Py-phase were collected at $133 \mathrm{GPa}$ for the sample synthesized from $\mathrm{Fe}_{2} \mathrm{O}_{3}$ in excess $\mathrm{H}_{2} \mathrm{O}$ and 53-81 GPa for the sample synthesized from $\mathrm{Fe}_{2} \mathrm{O}_{3}$ in the pure $\mathrm{O}_{2}$ medium.

X-ray Raman scattering experiments. XRS measurements on oxygen $K$-edge spectra of the Py-phase were conducted at $110 \mathrm{GPa}$ by using the XRS technique at beamline 16ID-D, APS, ANL. With the new $320 \times 400 \mathrm{~mm}$ IDT mirror, the incident monochromatic X-ray beam were focused down to 5-7 $\mu \mathrm{m}$ in FWHM at the sample position. With the sample in a diamond-anvil cell mounted on the rotation center of the XRS spectrometer, oxygen $K$-edge spectra were collected by scanning the incident beam energy from 518 to $578 \mathrm{eV}$ above the analyzer elastic scattering energy of $10.4467 \mathrm{keV}$, with an energy interval of $0.5 \mathrm{eV}$. The spectra were collected and averaged over $40 \mathrm{~h}$ of irradiation time. The Py-phase for the XRS measurements was synthesized from $\mathrm{FeOOH}$ (goethite) at $110 \mathrm{GP}$ and 2000-2200 K using laser-heating systems at 13ID-D and HPSynC. LiF platelets were deliberately used as pressure-transmitting medium and thermal insulating layers. LiF does not contain any oxygen and would not contribute any signals to XRS measurements on oxygen $K$-edge spectra of the Py-phase.

X-ray emission spectroscopy experiments. High-pressure XES measurements of the Py-phase at $300 \mathrm{~K}$ were performed at beamline 16-IDD, APS, ANL using a Rowland circle configuration with a spherical silicon (4 40 ) analyzer crystal with resolution of $\sim 1 \mathrm{eV}$. A helium tube was used to reduce scattering by air. An incident X-ray beam with an energy of $11.3 \mathrm{keV}$ was used to excite the emission. The collection time for each XES spectrum was $\sim 1 \mathrm{~h}$. The $4-7$ spectra were added for good statistics at a given pressure. Raman spectra of the diamond anvils were collected as a pressure gauge before and after each measurement while the ruby scale was cross-checked below $60 \mathrm{GPa}$. The Py-phase samples were synthesized from $\mathrm{Fe}_{2} \mathrm{O}_{3}$ and $\mathrm{H}_{2} \mathrm{O}$ approximately at $100-110 \mathrm{GPa}$ and $2000 \mathrm{~K}$. The XES spectrum of the Py-phase at the highest pressure of $133( \pm 4)$ GPa was used as the reference for the low-spin state. The total spin moment was then evaluated using the integrated spectral area from $7030 \mathrm{eV}$ to $7048 \mathrm{eV}$ with respect to that of the low-spin reference (Supplementary Figure 9).

Theoretical simulations. Now with experimental knowledge of materials as the base, theory can successfully provide accurate and quantitative details in the extended pressure-temperature regime inaccessible to experiment ${ }^{54}$. The structure of $\mathrm{FeO}_{2}$ has attracted many computational studies. However, an accurate description about its electron-correlation part in calculating the total energy still remains a challenging task. Based on previous studies, $\mathrm{O} 2 p$ orbitals may hybrid with Fe $3 d$ orbitals at the Fermi surface. It may cause $\mathrm{O}$ electrons to contribute to the electron-correlation portion in calculating the total energy. We note the linearresponse method is excellent in dealing with the exchange-correlation energy for one atom species (e.g. Fe), although it may encounter issues for two types of atoms at the same time. Therefore, the use of Hubbard parameter $U$ gives a first-order approximation to this energy portion. Combined with the self-consistent linearresponse approach ${ }^{55,56}$, many experimental observations can be reproduced by theory.

First-principles calculations based on density function theory using the package VASP were carried out using the projector-augmented wave pseudopotential and a plane-wave cutoff energy of $600 \mathrm{eV}$. The exchangecorrelation functional employs the parameterization by Perdew, Burke, and Ernzerhof under the Generalized Gradient Approximation. We follow our previous computational recipe ${ }^{18}$ to approximate the on-site Coulomb interaction by the Hubbard method $(U=5.0$ and $J=0.8 \mathrm{eV})$. In parallel, we also employed the Heyd-Scuseria-Ernzerhof screened hybrid functional (HSE) that was recently adopted for a variety of iron-oxides and calculated reliable structural, magnetic, and electronic properties ${ }^{57}$. For HSE-type functional, the screening variable was set to 0.15 since it produces reasonable values in other $\mathrm{Fe}-\mathrm{O}$ minerals like wüstite, magnetite, hematite, and goethite. We generated $k$ point in gamma centered Monkhorst-Pack grids of $7 \times 7 \times 7$ throughout our simulation. The structures were relaxed for atomic position, cell shape, and volumes for target pressures until the force acting on each atom was smaller than $0.01 \mathrm{eV} / \AA$. Following the Bader charge division scheme, we are able to determine the valence state of $\mathrm{Fe}$ and $\mathrm{O}$ atoms from the electron charge density (Table S1). Calculated valence states definitely are against ferric $\left(\mathrm{Fe}^{3+}\right)$ or ferryl $\left(\mathrm{Fe}^{4+}\right)$ state of iron. Iron and oxygen atoms in pyrite-structured $\mathrm{FeO}_{2}$ exhibit partial covalent feature, which is recognized as a common feature in pyrite $\mathrm{FeS}_{2}$ model $^{37}$. Therefore, our simulation results confirm that the valence state of iron in the Py-phase is close to $\mathrm{Fe}^{2+}$ with $\mathrm{O}-\mathrm{O}$ bonding.

\section{Data availability}

The datasets generated during and/or analysed during the current study are available from the corresponding authors and J.L. (Jin.Liu@hpstar.ac.cn).

Received: 25 April 2018 Accepted: 12 December 2018

Published online: 11 January 2019

\section{References}

1. Stagno, V., Ojwang, D. O., McCammon, C. A. \& Frost, D. J. The oxidation state of the mantle and the extraction of carbon from Earth's interior. Nature 493, 84-88 (2013)

2. Bykova, E. et al. Structural complexity of simple $\mathrm{Fe}_{2} \mathrm{O}_{3}$ at high pressures and temperatures. Nat. Commun. 7, 10661 (2016).

3. Lavina, B. et al. Discovery of the recoverable high-pressure iron oxide $\mathrm{Fe}_{4} \mathrm{O}_{5}$. Proc. Natl Acad. Sci. USA 108, 17281-17285 (2011).

4. Lavina, B. \& Meng, Y. Unraveling the complexity of iron oxides at high pressure and temperature: synthesis of $\mathrm{Fe}_{5} \mathrm{O}_{6}$. Sci. Adv. 1, e1400260 (2015).

5. Badro, J. et al. Electronic transitions in perovskite: possible nonconvecting layers in the lower mantle. Science 305, 383-385 (2004).

6. Lin, J.-F., Speziale, S., Mao, Z. \& Marquardt, H. Effects of the electronic spin transitions of iron in lower mantle minerals: implications for deep mantle geophysics and geochemistry. Rev. Geophys. 51, 244-275 (2013).

7. Deng, J. \& Lee, K. K. M. Viscosity jump in the lower mantle inferred from melting curves of ferropericlase. Nat. Commun. 8, 1997 (2017).

8. Gihan, L. W., Chris, J. P. \& Needs, R. J. Computational searches for iron oxides at high pressures. J. Phys.: Condens. Matter 27, 455501 (2015).

9. $\mathrm{Hu}, \mathrm{Q}$. et al. $\mathrm{FeO}_{2}$ and $\mathrm{FeOOH}$ under deep lower-mantle conditions and Earth's oxygen-hydrogen cycles. Nature 534, 241-244 (2016).

10. $\mathrm{Hu}, \mathrm{Q}$. et al. Dehydrogenation of goethite in Earth's deep lower mantle. Proc. Natl Acad. Sci. USA 114, 1498-1501 (2017). 
11. Nishi, M., Kuwayama, Y., Tsuchiya, J. \& Tsuchiya, T. The pyrite-type highpressure form of FeOOH. Nature 547, 205-208 (2017).

12. Boulard, E. et al. $\mathrm{CO}_{2}$-induced destabilization of pyrite-structured $\mathrm{FeO}_{2} \mathrm{H} x$ in the lower mantle. Natl Sci. Rev. 0, 1-8 (2018).

13. Mao, H. K. et al. When water meets iron at Earth's core-mantle boundary. Natl Sci. Rev. 4, 870-878 (2017).

14. Liu, J. et al. Hydrogen-bearing iron peroxide and the origin of ultralowvelocity zones. Nature 551, 494-497 (2017).

15. Yuan, L. et al. Chemical reactions between $\mathrm{Fe}$ and $\mathrm{H}_{2} \mathrm{O}$ up to megabar pressures and implications for water storage in the Earth's mantle and core. Geophys. Res. Lett. 45, 1330-1338 (2018).

16. Lyons, T. W., Reinhard, C. T. \& Planavsky, N. J. The rise of oxygen in Earth's early ocean and atmosphere. Nature 506, 307-315 (2014).

17. Streltsov, S. V., Shorikov, A. O., Skornyakov, S. L., Poteryaev, A. I. \& Khomskii, D. I. Pyrite $\mathrm{FeO}_{2}$ : material "in between"' oxides and peroxides. Sci. Rep. 7, 13005 (2017).

18. Jang, B. G., Kim, D. Y. \& Shim, J. H. Metal-insulator transition and the role of electron correlation in $\mathrm{FeO}_{2}$. Phys. Rev. B 95, 075144 (2017).

19. Shen, G. \& Mao, H. K. High-pressure studies with $\mathrm{x}$-rays using diamond anvil cells. Rep. Prog. Phys. 80, 016101 (2017).

20. Wilke, M., Farges, F., Petit, P. E., Brown, G. E. \& Martin, F. Oxidation state and coordination of Fe in minerals: an Fe K-XANES spectroscopic study. Am. Mineral. 86, 714-730 (2001).

21. Calas, G. \& Petiau, J. Coordination of iron in oxide glasses through highresolution K-edge spectra: information from the pre-edge. Solid State Commun. 48, 625-629 (1983).

22. Sanson, A. et al. Local structure and spin transition in $\mathrm{Fe}_{2} \mathrm{O}_{3}$ hematite at high pressure. Phys. Rev. B 94, 014112 (2016).

23. Lee, S. K., Eng, P. J. \& Mao, H. K. in Spectroscopic Methods in Mineralogy and Materials Science Vol. 1 Reviews in Mineralogy \& Geochemistry (Grang S. Henderson, Daniel R. Neuville, \& Robert T. Downs eds.) Ch. 4, 139-174 (Mineralogical Society of America, Chantilly, Virginia 2014).

24. Meng, Y. et al. Inelastic X-ray scattering of dense solid oxygen: evidence for intermolecular bonding. Proc. Natl Acad. Sci. USA 105, 11640-11644 (2008).

25. Sturhahn, W. CONUSS and PHOENIX: evaluation of nuclear resonant scattering data. Hyperfine Interact. 125, 149-172 (2000).

26. Pasternak, M. P. et al. High pressure collapse of magnetism in $\mathrm{Fe}_{0.94} \mathrm{O}$ : Mössbauer spectroscopy beyond 100 GPa. Phys. Rev. Lett. 79, 5046-5049 (1997).

27. Solomatova, N. V. et al. Equation of state and spin crossover of $(\mathrm{Mg}, \mathrm{Fe}) \mathrm{O}$ at high pressure, with implications for explaining topographic relief at the coremantle boundary. Am. Mineral. 101, 1084-1093 (2016).

28. Lin, J.-F. et al. Synchrotron Mössbauer spectroscopic study of ferropericlase at high pressures and temperatures. Am. Mineral. 94, 594-599 (2009).

29. Dyar, M. D., Agresti, D. G., Schaefer, M. W., Grant, C. A. \& Sklute, E. C. Mössbauer spectroscopy of earth and planetary materials. Annu. Rev. Earth Planet. Sci. 34, 83-125 (2006).

30. Badro, J. et al. Iron partitioning in Earth's mantle: toward a deep lower mantle discontinuity. Science 300, 789-791 (2003).

31. Lavina, B. et al. Siderite at lower mantle conditions and the effects of the pressure-induced spin-pairing transition. Geophys. Res. Lett. 36, L23306 (2009).

32. Kuwayama, Y., Hirose, K., Sata, N. \& Ohishi, Y. Pressure-induced structural evolution of pyrite-type $\mathrm{SiO}_{2}$. Phys. Chem. Miner. 38, 591-597 (2011).

33. Shirako, Y. et al. Synthesis, crystal structure, and electronic properties of highpressure $\mathrm{PdF}_{2}$-type oxides $\mathrm{MO}_{2}(\mathrm{M}=\mathrm{Ru}, \mathrm{Rh}, \mathrm{Os}, \mathrm{Ir}, \mathrm{Pt})$. Inorg. Chem. 53, 11616-11625 (2014).

34. Hoffman, C., Ropp, R. \& Mooney, R. Preparation, properties and structure of cadmium peroxide. J. Am. Chem. Soc. 81, 3830-3834 (1959).

35. Lu, C., Amsler, M. \& Chen, C. Unraveling the structure and bonding evolution of the newly discovered iron oxide $\mathrm{FeO}_{2}$. Phys. Rev. B 98, 054102 (2018).

36. Henkelman, G., Arnaldsson, A. \& Jónsson, H. A fast and robust algorithm for Bader decomposition of charge density. Comput. Mater. Sci. 36, 354-360 (2006).

37. Hu, J., Zhang, Y., Law, M. \& Wu, R. First-principles studies of the electronic properties of native and substitutional anionic defects in bulk iron pyrite. Phys. Rev. B 85, 085203 (2012).

38. Zhu, S.-c, Hu, Q., Mao, W. L., Mao, H. K. \& Sheng, H. Hydrogen-bond symmetrization breakdown and dehydrogenation mechanism of $\mathrm{FeO}_{2} \mathrm{H}$ at high pressure. J. Am. Chem. Soc. 139, 12129-12132 (2017).

39. Lundegaard, L. F., Weck, G., McMahon, M. I., Desgreniers, S. \& Loubeyre, P. Observation of an $\mathrm{O}_{8}$ molecular lattice in the $\varepsilon$ phase of solid oxygen. Nature 443, 201-204 (2006)

40. McCammon, C. The paradox of mantle redox. Science 308, 807-808 (2005).
41. Cavazzoni, C. et al. Superionic and metallic states of water and ammonia at giant planet conditions. Science 283, 44-46 (1999)

42. Goncharov, A. F., Struzhkin, V. V., Somayazulu, M., Hemley, R. J. \& Mao, H. $\mathrm{K}$. Compression of ice to $210 \mathrm{GPa}$ : evidence for a symmetric hydrogen bonded phase. Science 273, 218-220 (1996).

43. Freiman, Y. A., Jodl, H. J. \& Crespo, Y. Solid oxygen revisited. Phys. Rep. 743, 1-56 (2018).

44. McCammon, C. Perovskite as a possible sink for ferric iron in the lower mantle. Nature 387, 694-696 (1997).

45. Millot, M. et al. Experimental evidence for superionic water ice using shock compression. Nat. Phys. 14, 297-302 (2018).

46. Irifune, T. et al. Iron partitioning and density changes of pyrolite in Earth's lower mantle. Science 327, 193-195 (2010).

47. Nomura, R. et al. Spin crossover and iron-rich silicate melt in the Earth's deep mantle. Nature 473, 199-202 (2011).

48. Zhang, L., Yuan, H., Meng, Y. \& Mao, H. K. Discovery of a hexagonal ultradense hydrous phase in (Fe,Al)OOH. Proc. Natl Acad. Sci. USA 115, 1720510115 (2018).

49. Prakapenka, V. B. et al. Advanced flat top laser heating system for high pressure research at GSECARS: application to the melting behavior of germanium. High Press. Res. 28, 225-235 (2008).

50. Meng, Y., Hrubiak, R., Rod, E., Boehler, R. \& Shen, G. New developments in laser-heated diamond anvil cell with in situ synchrotron $\mathrm{x}$-ray diffraction at High Pressure Collaborative Access Team. Rev. Sci. Instrum. 86, 072201 (2015).

51. Fei, Y. et al. Toward an internally consistent pressure scale. Proc. Natl Acad. Sci. USA 104, 9182-9186 (2007).

52. Sturhahn, W. Nuclear resonant spectroscopy. J. Phys.: Condens. Matter 16, S497-S530 (2004)

53. Akahama, Y. \& Kawamura, H. Pressure calibration of diamond anvil Raman gauge to 410 GPa. J. Phys.: Conf. Ser. 215, 012195 (2010).

54. Wu, Z., Justo, J. F. \& Wentzcovitch, R. M. Elastic anomalies in a spincrossover system: ferropericlase at lower mantle conditions. Phys. Rev. Lett. 110, 228501 (2013).

55. Cococcioni, M. \& de Gironcoli, S. Linear response approach to the calculation of the effective interaction parameters in the LDA+U method. Phys. Rev. B 71, 035105 (2005).

56. Kulik, H. J., Cococcioni, M., Scherlis, D. A. \& Marzari, N. Density functional theory in transition-metal chemistry: a self-consistent hubbard $U$ approach. Phys. Rev. Lett. 97, 103001 (2006).

57. Meng, Y. et al. When density functional approximations meet iron oxides. J. Chem. Theory Comput. 12, 5132-5144 (2016).

58. Lin, J.-F. et al. Phonon density of states of $\mathrm{Fe}_{2} \mathrm{O}_{3}$ across high-pressure structural and electronic transitions. Phys. Rev. B 84, 064424 (2011).

\section{Acknowledgements}

We thank A. Shahar for providing the ${ }^{57} \mathrm{Fe}$-enriched hematite $\left(\mathrm{Fe}_{2} \mathrm{O}_{3}\right.$ with ${ }^{57} \mathrm{Fe}$ of $>96.5 \%$ ) powder and $\mathrm{X}$. Wu for $\mathrm{CaFeO}_{3}$ samples. We acknowledge E.E. Alp, C. KenneyBenson, T. Gu, and E. Greenberg for experimental assistance and helpful discussions. SMS, XAS, XES, and XRD measurements were performed at the High Pressure Collaborative Access Team (HPCAT 16-IDB and 16-IDD), APS, ANL. HPCAT operations are supported by DOE-NNSA's Office of Experimental Sciences. Y.X., P.C., and Y.M. acknowledge the support of DOE-BES/DMSE under Award DE-FG02-99ER45775. A portion of the XRD experiments was performed at GeoSoilEnviroCARS (Sector 13ID-D) at the APS, ANL. GeoSoilEnviroCARS is supported by the National Science Foundation -Earth Sciences (EAR-1128799) and Department of Energy-Geosciences (DE-FG0294ER14466). The Advanced Photon Source is a U.S. Department of Energy (DOE) Office of Science User Facility operated for the DOE Office of Science by Argonne National Laboratory under Contract No. DE-AC02-06CH11357. The computational work was conducted on the SR10000-K1/52 supercomputing facilities of the Institute for Materials Research, Tohoku University. W.L.M. and J.L. acknowledge support from the NSF Geophysics Program (EAR 1446969) and the Deep Carbon Observatory. H.K.M. and Q.H. were supported by NSF Grants EAR-1345112, EAR-1722515, and EAR-1447438. This work was also partially supported by the National Natural Science Foundation of China (Grant no. U1530402).

\section{Author contributions}

J.L., Q.H., L.Y., Y.X., P.C., Y.M., and V.B.P. carried out the experiment. J.L., W.B., Q.H., W.L.M., and H.-K.M. performed the experimental data analysis and interpretation. Q.H. performed the theoretical simulation. J.L., W.L.M., and H.-K.M. conceived and designed the project. J.L., Q.H., W.L.M., and H.-K.M. wrote the manuscript. All authors contributed to the discussion of the results and revision of the manuscript. 


\section{Additional information}

Supplementary Information accompanies this paper at https://doi.org/10.1038/s41467018-08071-3.

Competing interests: The authors declare no competing interests.

Reprints and permission information is available online at http://npg.nature.com/ reprintsandpermissions/

Journal peer review information: Nature Communications thanks the anonymous reviewers for their contributions to the peer review of this work. Peer reviewer reports are available.

Publisher's note: Springer Nature remains neutral with regard to jurisdictional claims in published maps and institutional affiliations. (c) Open Access This article is licensed under a Creative Commons Attribution 4.0 International License, which permits use, sharing, adaptation, distribution and reproduction in any medium or format, as long as you give appropriate credit to the original author(s) and the source, provide a link to the Creative Commons license, and indicate if changes were made. The images or other third party material in this article are included in the article's Creative Commons license, unless indicated otherwise in a credit line to the material. If material is not included in the article's Creative Commons license and your intended use is not permitted by statutory regulation or exceeds the permitted use, you will need to obtain permission directly from the copyright holder. To view a copy of this license, visit http://creativecommons.org/ licenses/by/4.0/.

(C) The Author(s) 2019 